\title{
Measuring misconceptions about traumatic brain injury: Are existing scales misconceived?
}

Eleanor Bryant ${ }^{\mathrm{a}}$, Dr Claire Williams ${ }^{\mathrm{a} *}$, Dr Ruth Horry ${ }^{\mathrm{a}}$, and Professor Andrew Worthington ${ }^{\text {abc }}$

${ }^{a}$ Department of Psychology, Swansea University, Swansea, United Kingdom; ${ }^{b} H e a d w i s e$, Birmingham, United Kingdom; 'Swansea University Medical School, Swansea University, Swansea, United Kingdom.

*Corresponding Author:

Dr Claire Williams, Department of Psychology, College of Human and Health Sciences,

Swansea University, Singleton Park, Swansea, SA2 8PP, Wales, UK.

Tel: (+44) 1792295629

Email Address: claire.williams@swansea.ac.uk OCRID: https://orcid.org/0000-0002-0791-744XX

Twitter: DrCWilliams1

Abstract Word Count: 173 words.

Manuscript Word Count (excluding title page, abstract, references, tables, figures): 5230 


\section{Measuring misconceptions about traumatic brain injury: Are existing scales misconceived?}

Primary Objectives: To conduct a review of the misconception literature relating to traumatic brain injury (TBI) and to critically review the comprehensiveness, psychometric properties and other qualities of existing scales designed to measure knowledge and misconceptions of TBI.

Methods: Terms relating to misconceptions, misconception scales, public perceptions and traumatic brain injury were used to identify existing scales. The initial search was expanded using the reference lists and citations of relevant articles.

Main Outcomes: Eight scales were identified for full review, with several sharing a common set of items. The majority of scales were designed to measure public perceptions of TBI, although some were developed for use in specific populations (e.g. sports, professional samples). Existing scales are limited by their scope and breadth of coverage, adoption of a medicalised view of TBI, scaling and scoring issues, failure to use a conceptual framework, and by numerous psychometric issues related to reliability and validity.

Conclusions: There are a number of weaknesses attached to existing scales. Several recommendations are made to promote and inform future scale development.

Keywords: traumatic brain injury; public perceptions; misconceptions; measurement; validity; psychometric 


\section{Introduction}

With an estimated sixty-nine million new cases each year, traumatic brain injury (TBI) is a serious global public health issue (1). In the UK alone, 1.3 million people are estimated to be living with TBI related disability at any one time (2) with a reported $10 \%$ increase in incidence over the last decade alone (3). Typically more prevalent in males (4), among people aged $<25$ years and $>75$ years $(5,6)$, and in incarcerated groups $(7,8)$, TBI is often described as a 'silent epidemic’ as its effects are often invisible and underestimated (5).

Public awareness, knowledge and understanding of TBI is typically poor (9), with members of the general public often holding significant misconceptions about the physical and psychosocial sequelae of TBI (10-12). Gouvier et al. (9) found that $46 \%$ of 221 community participants believed that a second blow to the head could reduce amnesia and restore lost memories, and Hux et al. (10) reported that $93.4 \%$ of their general population sample believed that a person with a brain injury can have a memory impairment so severe that they cannot recognise family members or remember autobiographical events, but can be normal in every other way. Similar misconceptions have also been demonstrated in populations who provide services to those with TBI, including those in the medical, educational, correctional, and healthcare sectors (13-16). Swift and Wilson (17) found that medical professionals without specialist brain injury knowledge held numerous misconceptions around the complexity of the recovery process. Similarly, Linden et al. (18) found that $74 \%$ of their sample of educational professionals did not know or incorrectly agreed with the statement 'children who are knocked unconscious wake up quickly with no lasting effects', and Yuhasz (16) found an overall TBI misconception rate of $24 \%$ (ranging from $0 \%-73 \%$ for individual items) in a sample of health professionals working in a correctional setting. 
Various factors have been linked to knowledge, expectations, and beliefs about TBI, including both demographic (e.g. age, gender) and experiential factors (e.g. level of education, occupation), as well as passive (e.g. via information in the media) and active (e.g. direct exposure to TBI) cognitive processes $(10,19,20)$. Surprisingly, however, personal exposure to TBI has been inconsistently related to accuracy of TBI knowledge $(9,16,21)$, and even when people report receiving information from health professionals, knowledge of TBI recovery and related sequelae is still poor (9). In contrast, media misinformation has been frequently cited as a source of misconceptions for members of the lay public (e.g. Hux et al. (10)). For instance, the media often inaccurately portray a second injury as curative or use memory loss as a plot device rather than realistically portraying the full breadth of changes that can occur after TBI $(19,22)$.

Understanding misconceptions, awareness and knowledge of TBI is critical in reducing stigmatisation and subsequent discrimination of survivors with TBI. Moreover, raising awareness of the hidden and often invisible nature of TBI (23) is pivotal for creating accurate and realistic expectations around TBI and the recovery process. Indeed, a disconnect often exists between what is expected of a person after TBI once an apparently good physical and external recovery has been achieved and the daily challenges they actually face (17). For instance, returning to the workplace and integrating back into the community can often be a struggle after TBI $(24,25)$ irrespective of whether there is an accompanying outward manifestation of injury. Tackling such misconceptions requires a multi-level approach in the form of public awareness campaigns and training healthcare, correctional, and educational professionals to recognise the hidden impact of TBI. Working alongside survivors and their families directly is also essential. Rehabilitation programmes can endeavour to understand what misconceptions survivors experience within their communities, and work on strategies 
for dealing with them to promote more positive outcomes (23). However, in order to achieve this, appropriate methods of examining misconceptions and awareness of TBI must first be available.

Several scales have been developed to measure misconceptions about TBI, but as we will argue, these scales are often narrow in their focus, failing to capture many relevant domains of knowledge (e.g. injury invisibility, psychosocial outcomes), are often inconsistently administered across studies, and have frequently not been subjected to thorough psychometric evaluation (19). Through reviewing the comprehensiveness and psychometric properties of existing scales, we will highlight the need to develop a valid and comprehensive tool for the measurement of TBI misconceptions which has a more robust and conceptually driven approach to its development. We do not claim that the scales reviewed here comprise an exhaustive compendium of all available measures. Rather, we have focussed on the scales that are in widespread use, rendering them worthy of review. A brief description of each of these scales is presented followed by a critical review of their construction, psychometric properties, and use in research.

\section{Review of existing scales - perceptions and misconceptions of TBI}

Here we briefly describe existing scales, outlining their development, format, structure, content, and application in research.

The Common Misconceptions about Head Injury and Recovery Survey

Gouvier et al. (9) developed a 25-item survey to capture the types of misconceptions family members commonly hold about TBI when their loved ones commence rehabilitation. Rated on a 4-point Likert scale of 'true,' 'probably true' 'probably false' and 'false', items cover five 
core domains: (1) 'use of seat belts' (e.g. 'Wearing seatbelts causes as many injuries as it prevents'); (2) ‘nature of unconsciousness’ (e.g. 'When people are knocked unconscious, most wake up shortly with no lasting effects'); (3) 'amnesia’ (e.g. 'People can forget who they are and not recognize others, but be normal in every other way'); (4) 'characteristics associated with brain injury’ (e.g. 'Emotional problems after head injury are usually not related to brain damage'), and (5) 'recovery from brain injury’ (e.g. 'Once a recovering person feels "back to normal” the recovery process is complete'). Originally completed by 221 members of the public in a US shopping centre, misconceptions were found across each item and domain (misconception endorsements ranged from 11.31\% - 82.35\%), with at least half of respondents endorsing misconceptions in both the 'Amnesia' and 'Recovery' domains. For instance, 82.35\% and $73.76 \%$ of respondents significantly endorsed the statements 'People can forget who they are and not recognize others, but be normal in every other way' and 'People who have had one head injury are more likely to have a second one', respectively.

Since its initial development, the Gouvier et al. (9) survey has formed the basis of several subsequent scales (see Table 1). For instance, Willer et al. (12) utilised nine items to investigate misconception rates in 313 members of the public from different regions across the USA, whilst Guilmette and Paglia (21) used 11 of the original items, plus eight new forensically orientated items to explore misconception rates in an urban setting. Overall, misconceptions rates were similar across the three studies, although Guilmette and Paglia (21) found a lower misconception rate for two concussion items.

\section{Insert table 1 here}




\section{Brain Injury Misconception Scale (BIMS)}

To examine the impact of public health awareness campaigns and to explore misconception rates over time, Hux et al. (10) selected 17 items which had shown misconception rates of at least 25\% from Gouvier et al.’s (9) and Willer et al.’s (12) research. Collectively, these items formed the Brain Injury Misconception Scale (BIMS) (26). Hux et al (10) altered the wording of some items to improve comprehension and to reflect changes in language acceptability (e.g. the word 'retarded' was replaced with 'disabled'). Using a two point true-false response format, four domains were captured: (1) general knowledge (e.g. 'Emotional problems after head injury are usually not related to brain damage’); (2) coma and unconsciousness (e.g. 'When people are knocked unconscious, most wake up shortly with no lasting effects'); (3) memory deficits (e.g. 'People with amnesia for events before the injury usually have trouble learning new things too'), and (4) recovery (e.g. 'It is good advice to remain inactive during recovery'). Hux et al. (10) reported lower misconception rates than both Gouvier et al. (9) and Willer et al. (12) in the general knowledge domain, potentially attributable to improved knowledge following public awareness campaigns. However, significant misconceptions remained in the coma, memory deficits and recovery domains.

Chapman and Hudson (27) also found evidence of misconceptions on the BIMS in a UK sample of 332 people. Compared to Hux et al. (10) and their USA sample, UK respondents reported significantly more misconceptions in the general knowledge, memory deficits, and coma domains. However, given the different sampling strategies adopted across studies (i.e. face-to-face survey versus snowball strategy via email, post or in-person) it is difficult to draw firm conclusions about these differences. 
Common Misconceptions of Traumatic Brain Injury (CM-TBI)

Consisting of 40 items (24 adapted from Gouvier et al. (9)) rated on a four-point response format (true, probably true, probably false, or false), the CM-TBI (28) covers: (1) 'seatbelts/prevention'; (2) 'brain damage’; (3) 'unconsciousness’; (4) 'amnesia’; (5) 'recovery’; (6) ‘rehabilitation', and (7) ‘brain injury sequelae’. Items were generated through clinical experience of observing the information, knowledge and misperceptions that family and survivors hold in relation to brain injury. When administered to 51 family members of TBI survivors at their point of entry into inpatient rehabilitation services (28), an overall misconception rate of $23.1 \%$ was reported, with misconceptions particularly evident in the amnesia, recovery and unconsciousness domains.

McKinley and Buck (29) adapted the CM-TBI to assess educators’ knowledge of brain injury by excluding items measuring concussion knowledge, choosing instead to use an adapted 20-item concussion awareness questionnaire (30). Similarly, Farmer and Johnson-Gerard (31) slightly modified the wording of the original 40-item CM-TBI (e.g. substituting 'people/person' to 'child/children'), to assess educators' ( $n=184)$ versus rehabilitation specialists $(\mathrm{n}=111)$ knowledge of childhood TBI. Across both studies, comparable misconception rates were seen in half of the common survey items, although McKinlay \& Buck (29) found fewer misconceptions on $37 \%$ of the items, particularly in relation to recovery, and higher misconception rates on $17 \%$ of items, with some measuring emotional changes after brain injury. 


\section{Head Injury Knowledge Scale (HIKS)}

The HIKS (26) attempts to capture how individuals may simultaneously fail to recognise (minimisation) common outcomes (e.g. 'have trouble remembering details of recent conversations') and overgeneralise by endorsing outcomes that are not commonly associated with TBI (e.g. 'become upset and yell for no reason'). A true response in the 'overgeneralisation' and a false response in the 'minimisation' domains indicate the presence of a misconception. During its development, 13 individuals with TBI, screened for a good level of self-awareness and insight, completed the scale. Only items that were widely agreed upon were retained. 101 participants without TBI then completed the HIKS and BIMS; the latter was included so that the convergent validity of the HIKS could be examined. However, the internal consistency of the BIMS was found to be extremely low $(\alpha=.14)$, undermining its credibility as a suitable tool for validating the HIKS. Even so, misconceptions rates for individual items ranged from $11.11 \%$ - 57.58\%, with participants more likely to over-generalise than minimise the effects of TBI. However, females overgeneralised more than males, and participants with direct experience of TBI tended to overgeneralise less than those with indirect/limited experience.

\section{Brain Injury and Schizophrenia Awareness Scale (BISAS)}

The BISAS (32) aims to capture public understanding of the distinct and common functional outcomes of schizophrenia and brain injury. Consisting of 31-items in total, 12 items relate to the shared outcomes of brain injury and schizophrenia (e.g. 'have a poor understanding of the effects of their condition', 9 to the common and distinct outcomes of TBI (e.g. 'Have difficulty scanning the environment to find an object they are looking for'), 6 to the common and distinct outcomes of schizophrenia (e.g. 'Believe they are highly influential and have special gifts'), 
and 4 items which are generally unrelated to either (e.g. 'Consistently complete tasks from start to finish'). A 4-point response scale allows respondents to indicate if they believe items are common to TBI or schizophrenia, are common to both, or are 'rarely experienced' in either condition. Participants ( $n=175$, lay sample) were generally more accurate in identifying the distinct effects of TBI and schizophrenia rather than their shared impacts - though this pattern was not found in a professional sample made up of 40 individuals working in the field of mental health or for disability services. Overall, behavioural and emotional consequences were more likely to be attributed to schizophrenia and conversely, cognitive deficits were perceived as more common after TBI in the lay sample.

\section{Rosenbaum Concussion Knowledge and Attitudes Survey (RoCKAST-ST)}

Scales have also been developed for use in specific contexts, such as the College Football Head Injury Survey (33) and the Knowledge and Attitudes about Sports Concussion Questionnaire (34). However, such measures have been heavily criticised for lacking psychometric integrity and for focusing on return to play without assessing wider perceptions of head injury (35). To address such limitations, Rosenbaum and Arnett (35) developed the RoCKAST-ST, a 55-item scale assessing knowledge of the causes and consequences of concussion, as well as attitudes towards 'return to play.' Items were drawn from a mixture of sources (e.g. Gouvier et al. (9)), and two composite scores can be calculated - a 'Concussion Knowledge Index' (CKI - score range 0-25) and a 'Concussion Attitudes Index’ (CAI - score range 15-75). The RoCKAST-ST has been used to survey 26 professional Champions League players in England (36) with findings highlighting how favourable attitudes towards safe play and/or good concussion knowledge do not necessarily translate into commitments towards safer behaviours on the pitch. 
Surveys developed for professional contexts

Surveys have also been developed to assess knowledge and perceptions of educators, who may, within their role, work alongside individuals with TBI. Hux et al. (13) developed a 65-item survey specifically designed for Speech and Language Pathologists, focusing on their perceived ability to facilitate the assessment, intervention and reintegration of children back into a classroom setting post-TBI. Covering legislative knowledge, TBI outcomes and training received, items are rated on a combination of yes/no and 5-point Likert response formats.

Similarly, the 'Perceptions of Brain Injury Survey’ (PBIS) assesses nurses’ beliefs and knowledge of TBI within their professional context, as well as how they would seek information to inform their practice (15). The PBIS was developed by adapting some items from Hux et al. (13) and contains three sections: (1) 20 items, scaled from 1 (none) to 4 (expert) on knowledge around care planning and clinical guidelines; (2) 17 items scaled from 1 (strongly agree) to 4 (strongly disagree) focusing on understanding of prognosis, perceived consequences, nursing role, and role of knowledge in practice, and (3) a categorical 'tick all that apply’ question concerning how a nurse might seek information to improve their practice (e.g. ask a more experienced colleague). The survey was emailed to all registered nurses in hospital departments within a regional healthcare system, with 330 online responses captured from paediatric nurses (15). Paediatric nurses frequently endorsed inaccurate beliefs directly related to TBI care, such as the perceived usefulness of TBI injury classification (i.e. mild, moderate or severe) to inform care planning in the absence of other information about the patient. In turn, such beliefs could negatively impact on care provision and compromise the accuracy of information relayed to families about recovery (15). 


\section{Evaluation of existing scales}

To determine whether there is need to develop a more valid and comprehensive tool for the measurement of TBI knowledge and misconceptions, it is important to consider how items for the most commonly used scales described above were generated, what processes were applied to the resultant scales to ensure reliability and validity (see Table 2), and how survey responses have been scored and presented in research. By applying these evaluations systematically to the scales, and walking through the process of scale development, areas for improvement can be identified

\section{Insert Table 2 here}

\section{Item development}

To a greater or lesser extent, several existing scales included or adapted items from Gouvier et al. (9). However, even though these items were originally derived from knowledge-based misconceptions evident in family members of individuals with TBI, suggesting good validity, such a bottom-up approach to item generation could equally have constrained the breadth of item coverage. Expanding on this approach, Springer et al. (28) drew on clinical experience when generating additional items for the CM-TBI, thereby improving the content and face validity of the scale. However, such approaches to item generation ultimately lack reference to an overarching theoretical or conceptual framework, increasing the risk that important items/domains are excluded.

In contrast, the HIKS and BISAS both used functional outcomes, focusing on the physical, behavioural, sensory and cognitive impairments associated with TBI as underlying 
knowledge constructs to establish scale items (e.g. (26)). However, whilst such an approach uses an overarching framework for item generation, the focus on symptoms and dysfunction within the individual conforms to a more medicalised view of brain injury, thus narrowing item coverage. Consequently, when compared to the CM-TBI with its broader coverage (i.e. impairment, psychological impact, rehabilitation and recovery), the HIKS and BISAS arguably lack relevance and applicability to applied research. What is apparent is the need to move towards a more holistic assessment of perceptions of TBI which incorporates a broad range of domains (e.g. social recovery and risk factors). Key to this is the need to model item generation on a clear conceptual-theoretical framework which moves beyond a medical model (37).

\section{Content validity}

Central to scale development is the need to consider the extent to which items represents all facets of a given construct and have discriminatory value. However, few existing scales have addressed content validity as part of the development process. For those that have (e.g. Ono et al. (26), pilot testing has enabled refinement of measures prior to testing in larger samples, identifying items for removal which lack any discriminatory value and/or are ambiguous in their meaning. For example, the HIKS was initially validated in a small sample of individuals with brain injury $(n=13)$ to ensure items mapped across to the real-world consequences of TBI. This information was then used to exclude items with an endorsement rate below $60 \%$, resulting in the removal of two items. However, using this endorsement threshold could still have resulted in the inclusion of items attracting inconsistent and/or variable responses. Other scales have tested items with brain injury professionals, asking small samples to check items for accuracy and possible ambiguity (e.g. (12, 33)). However, exisiting measures tend to rely on a single approach, even though the adoption of multiple different approches (e.g. drawing 
from the outcomes litertature and phenomenological experinces of those with TBI and their carers in addition to the aforementioned methods) would facilitate better content validity.

\section{Construct validity}

Less extensively investigated, examination of construct validity has typically involved collecting data from a professional sample for comparison against a lay population (e.g. (31)), an approach which is useful for detecting key areas where perceptions are most inaccurate in lay populations. For example, the BISAS compared responses from an expert (e.g. disability and mental health workers) versus non-expert community sample (32). An additional method for establishing construct validity is to scrutinise items for socially biased responding (38). For instance, Rosenbaum and Arnott (35) found that scores on the RoCKAST-ST did not significantly correlate with the Social Desirability Scale (SDS) (39). In sum, future scale development should focus more systematically on evaluating construct validity and should aim to use multiple methods.

\section{Response formats}

There are inherent problems with how existing tools have been scaled. First, most tools have utilised 2- or 4- point true/false scales (e.g. CM-TBI, BIMS), commonly dichotomising responses for analysis (e.g. $(9,11))$. For example, re-scoring uncertain responding (e.g. 'possibly true' or 'possibly false' responses) captured on 4 -point scales as either 'true' or 'false'. Second, even though capturing a lack of knowledge is as beneficial as uncovering inaccurate perceptions; few existing scales include a mid-point option for participants to indicate that they do not know the correct response. Linden et al. (18) found that participants frequently used the 'don't know' response on the CM-TBI. In over half of the 40 items

measured, a notable proportion (at least 30\%) of the sample responded with uncertainty, with 
$70 \%$ of respondents making use of the 'don't know' option on one specific item ('Drinking alcohol usually affects a young person differently after a brain injury'). Likewise, even though the RoCKAST-ST included a 'neutral' mid-point for items examining attitudes to safe play, the knowledge index utilises a true/false response format (35). These findings highlight the caveats that need to be considered when interpreting findings from misconception surveys. How data from survey responses have been treated before analysis, and whether any neutral midpoints have been included in the scales, warrants attention before definitive conclusions are made regarding misconception rates.

\section{Research designs and analysis of findings}

Reporting of misconception rates have tended to be descriptive, focussing on frequency counts in order to draw comparisons across research studies (e.g. (10, 27)). Advancements in this area should move towards more robust research designs which allow for measurement between different groups (19), such as different levels of exposure and experience of TBI and their relationship to knowledge. Block et al. (19) also advocates for the inclusion of effect sizes to improve comparisons across studies. Devising tools which can be scaled to produce overall scale scores and individual domains scores would also facilitate this process, allowing for a more reliable assessment of knowledge without being susceptible to possible idiosyncrasies of individual items. In line with this recommendation, more recently developed measures, including the HIKS and RoCKAST-ST have done this. However, these scales are used fairly infrequently in misconception research even though attempts to scale more established measures have been largely unsuccesful. For instance, Linden et al. (18) found that only 20 of the original 40 items from the CM-TBI loaded onto factors to produce a unified scale with four sub-scales. 


\section{Other psychometric considerations}

Researchers who have imported items from existing scales have tended to use them without reporting or investigating their reliability and validity (e.g. $(10,21))$. Indeed, it was only through the focused development of a new measure, namely the HIKS, that the internal consistency of the BIMS (based in part on Gouvier et al. (9)) was evaluated and found to be extremely low ( $\alpha=.14$ ) (26). In contrast, the CM-TBI which is widely used in applied settings (e.g. $(18,38)$ ) has been shown to have good levels of internal consistency ranging from $\alpha=.75$

-.85 (18, 38, 39). The later revised 20 item CM-TBI also evidenced good levels of internal consistency $(\alpha=.77-.84)(18,38)$ but was more varied across the four sub-scales: Recovery (8 items) $-\alpha=.73$; Sequelae (6 items) $-\alpha=.81$; Insight (3 items) $-\alpha=.61$, and Hidden Injury (3 items) $-\alpha=.55(40)$.

A key issue is that many existing measures have been retrospectively scrutinised in applied research, rather than at the point of development. Additionally, some measures that use items from the BIMS and CM-TBI have presented their results alongside previous research for comparison (e.g. (10)) yet no reliability testing over time has been carried out (e.g. testretest reliability). Consequently, fluctuations in misconception rates across studies could simply reflect issues with scale reliability rather than indicating contextual or real-world differences over time. Indeed, the RoCKAST-ST is the only existing measure where test-retest reliability has been examined, with the CKI showing good consistency in responses (ICC = .79) but with the CAI falling below acceptable levels (ICC = .67) (35). However, the test-retest interval was only two days, raising the possibility that participants would have been able to recall their responses from the previous session and respond in line with their recollections (42). 
To address some of these issues, the HIKS was psychometrically evaluated during its initial development phase (26). However, in the initial pilot, internal consistency of the overall measure was found to be extremely low $(\alpha=.31)$ even though the overgeneralisation and minimisation subscales evidenced acceptable levels of internal consistency ( $\alpha=.65$ and $\alpha=$ .68, respectively). A negative correlation was also found between the two sub-scales, giving weight to the claim that they represent two opposing dimensions (26). In its original format, a true-false response format was adopted; however, to carry out confirmatory factor analysis (CFA) and improve levels of internal consistency, a 4-point scale was subsequently adopted (43). Notably, the Cronbach's alpha values for the two subscales improved: overgeneralisation $-\alpha=.84$, and minimisation $-\alpha=.81$. CFA also demonstrated a good fit to the original twodimensional model developed by Ono et al. (26), although a moderate co-variance was found between the factors (43).

Equally, even though the RoCKAST (35) adopted a more thorough approach to scale development, its focus on sports related concussion and attitudes in a sporting context, limits its applicability to general population studies. This is also an issue with other scales that have been developed for targeted populations (e.g. PBIS) (15). Finally, scales developed to survey non-expert professionals in early research have not focused on evaluating reliability and validity (e.g. Hux et al. (13)) but later research on the PBIS for example, has referred to the scale as having good psychometric properties (15).

\section{Future directions and recommendations}

From reviewing existing measures pertaining to measure misconceptions and perceptions of TBI, it is apparent that an ideal instrument has yet to be developed. However, given the 
complexities involved with measuring perceptions and knowledge of TBI, it may be an unreasonable expectation that any single measure will be entirely sufficient. Although, that is not to say that a better, more reliable, valid, and conceptually driven measure should not be developed. When approaching this task, we believe that the following recommendations should be considered.

First, we recommend that the constructs assessed by existing knowledge-based measures are revisited. Existing items generated to assess the knowledge construct of TBI have mostly been drawn through clinical experience of misconceptions or by using the functional consequences of brain injury as a starting point. However, such approaches are limited by their lack of overarching conceptual framework, with some important areas neglected as a result. For example, the CM-TBI and HIKS $(26,28)$ predominantly cover the cognitive and emotional consequences of brain injury, neglecting to incorporate items related to social recovery. Further, even though there is undoubtedly some commonality across measures (i.e., inferred from common domains, importation of items from existing scales); there is equally large variation, suggesting that researchers do not have a homogeneous view of what they are trying to assess. Instead, a more conceptually driven approach to item generation may enable the development of a more holistic and consistent assessment of perceptions relating to brain injury. One possibility is to adopt the bio-psychosocial framework captured by the revised International Classification of Impairments Diseases and Handicaps (ICIDH-2) (37), a method commonly used for classifying and understanding the functional and social disadvantage associated with brain injury. Adoption of this framework would capture the multiple layers of disability arising from brain injury, including impairments (e.g. memory loss), activities (e.g. motor, communication problems) and participation/social handicap (e.g. community and 
working life), as well as an assessment of environmental factors (e.g. social attitudes towards people with TBI).

Second, a new measure would benefit from Likert scaling (e.g. 5-7 response formats) with inclusion of a neutral midpoint response option to allow discrimination between a lack of knowledge of TBI and a misconception. Third, newly developed scales should be subjected to more thorough psychometric evaluation. In addition to internal consistency, other properties of reliability need to be routinely investigated (e.g. test-re-test) and information about construct and content validity should be determined. Likewise, measures should be subjected to robust statistical analysis to identify overall scale and sub-scale structures during the development phase. Importantly, this would increase the measures' subsequent utility in applied research by reducing the risk of individual item idiosyncrasies and fluctuations, and by allowing direct comparison of misconception rates between populations (e.g. lay versus professional samples) and across time.

Fourth, it is also important to consider the broad spectrum of knowledge and attitudes that the public hold about individuals with TBI. Focused attempts to develop measures specific to TBI have, to date, been constrained to measuring knowledge of TBI and do not endeavour to measure social attitudes towards individuals with TBI alongside. The development of such measures have potential to facilitate and improve our understanding of public perceptions, by placing the individual within their social context; something which is critical for understanding and empathising with how individuals' with TBI have to navigate their social experiences (17). Drawing inspiration from the Positive and Negative Affect Scale (PANAS) (44), Linden and Crothers (45) asked both a public and student sample to rate their level of agreement to a 20item measure of positive and negative attributes (e.g. 'People with brain injuries can be... 
violent/confident'). Whilst there were core differences between the groups in how they perceived individuals with brain injury, both groups held some negative views (45). However, no psychometric evaluation was carried out of these 20 items during the research. Other research evaluating attitudes to brain injury has found that nurses hold more prejudicial attitudes towards survivors when they are presented as being to blame for their brain injury (i.e. resulting from taking drugs) compared to being presented as blameless (i.e. resulting from an aneurysm) (46). Similarly, prejudicial attitudes and less desire for social interaction has also been reported in lay samples when survivors are deemed responsible for their brain injury (45, 46). However, the two scales adopted to explore attitudes in these studies (Prejudice Evaluation Scale, PES - and Social Interaction Scale - SIS) (49) were devised specifically for evaluating attitudes towards AIDS. These measures were presented alongside scenarios and included items of little relevance to brain injury (e.g. PES Item - 'Would you attend a party where X was preparing dinner?'). Given these findings, developing new tools specifically designed to explore attitudes in the context of brain injury is equally as important.

\section{Concluding remarks}

This review has attempted to clarify the core issues that relate to the measurement of knowledge and misconceptions of brain injury, concluding that existing scales are limited by their scope and breadth of coverage, failure to use a conceptual framework, and by their lack of psychometric evaluation. Important to this discussion is how the invisible nature of many TBI sequelae is an underlying factor in public misperception (23). Indeed, observers often expect a physical marker for brain injury, and in the absence of one, will misattribute behaviour and actions to other causes (50). Owing to this, those living with TBI often feel that their disability is underestimated and/or trivialised (17). With no outward physical marker, and their hidden cognitive difficulties underestimated, more is often expected of them socially. In essence, 
looking normal on the outside means being viewed as functioning 'normally' on the inside (17). These misattributions are considered a potential driving force for negative societal consequences often experienced by those with brain injury $(19,23,49)$ and highlights the important role that brain injury knowledge and misconception scales have in improving our understanding of public perceptions. We hope that the critical analysis presented here will generate interest amongst researchers and clinicians to develop better, more reliable, valid, and conceptually driven misconception measures to improve our understanding of how those with TBI are currently perceived in society. 
Bryant - Misconceptions about TBI

\section{Declaration of interest}

The information in this manuscript and the manuscript itself is new and original and is not currently under review by any other publication and has never been published either electronically or in print. The authors have no financial relationships or conflict of interest to disclose. There are no financial sources of support to declare. 


\section{References}

1. Dewan MC, Rattani A, Gupta S, Baticulon RE, Hung Y, Punchak M, et al. Estimating the global incidence of traumatic brain injury. J Neurosurery. 2018;1-18.

2. Parsonage M. Report: An economic analysis Traumatic brain injury and offending. London; 2016.

3. Menon DK, Bryant CM. Time for change in acquired brain injury. Lancet Neurol. 2019;18:28. Available from: https://intbir.nih.gov/

4. Cassidy JD, Carroll LJ, Peloso PM, Holst H Von, Holm L, Kraus J, et al. Incidence, risk factors and prevention of mild traumatic brain injury: results of the WHO collaborating centre task force on mild traumatic brain injury. J Rehabil Med. 2004;43:28-60.

5. Rusnak M. Traumatic brain injury: Giving voice to a silent epidemic. Nat Rev Neurol. 2013;9(4):186-7. Available from: doi:10.1038/nrneurol.2013.38

6. Peeters W, van den Brande R, Polinder S, Brazinova A, Steyerberg EW, Lingsma HF, et al. Epidemiology of traumatic brain injury in Europe. Acta Neurochir (Wien). 2015;157:1683-96.

7. Fazel S, Philipson J, Gardiner L, Merritt R, Grann M. Neurological disorders and violence: A systematic review and meta-analysis with a focus on epilepsy and traumatic brain injury. J Neurol. 2009;256:1591-602.

8. Scottish Prison Service/NHS Scotland. National Prisoner Healthcare Network: brain injury and offending. Edinburgh: 2016.

9. Gouvier WD, Prestholdt PH, Warner MS. A Survey of Common Misconceptions About Head Injury and Recovery. Arch Clin Neuropsychol. 1988;3:331-43.

10. Hux K, Schram CD, Goeken T. Misconceptions about brain injury: A survey replication study. Brain Inj. 2006;20(5):547-53.

11. Pappadis MR, Sander AM, Struchen MA, Leung P, Smith DW. Common 
misconceptions about traumatic brain injury among ethnic minorities with TBI. J Head Trauma Rehabil. 2011;26(4):301-11.

12. Willer B, Johnson WE, Rempel RG, Linn R. A note concerning misconceptions of the general public about brain injury. Arch Clin Neuropsychol. 1993;8(5):461-5.

13. Hux K, Walker M, Sanger DD. Traumatic Brain Injury: Knowledge and SelfPerceptions of School Speech-Language Pathologists. Lang Speech Hear Serv Sch. 1996;27:172-84.

14. Oyesanya TO, Snedden TR. Pediatric nurses’ perceived knowledge and beliefs of evidence-based practice in the care of children and adolescents with moderate-to-severe traumatic brain injury. J Spec Pediatr Nurs. 2018;23.

15. Oyesanya TO, Thomas MA, Brown RL, Turkstra LS. Nurses’ Beliefs About Caring for Patients With Traumatic Brain Injury. West J Nurs Res. 2016;38(9):1114-38.

16. Yuhasz JE. Misconceptions About Traumatic Brain Injury Among Correctional Health Care Professionals. J Correct Heal Care. 2013;19(2):135-43.

17. Swift TL, Wilson SL. Misconceptions about brain injury among the general public and non-expert health professional: an exploratory study. Brain Inj. 2001;15(2):149-65.

18. Linden MA, Braiden HJ, Miller S. Educational professionals' understanding of childhood traumatic brain injury. Brain Inj. 2013;27(1):92-102.

19. Block CK, West SE, Goldin Y. Misconceptions and Misattributions About Traumatic Brain Injury: An Integrated Conceptual Framework. Am Acad Phys Med Rehabil. 2016;8:58-68.

20. Ernst WJ, Trice AD, Gilbert JL, Potts H. Misconceptions about traumatic brain injury and recovery among nursing students. J Head Trauma Rehabil. 2009;24(3):213-20.

21. Guilmette TJ, Paglia MF. The public's misconceptions about traumatic brain injury: A follow up survey. Arch Clin Neuropsychol. 2004;19:183-9. 
22. Baxendale S. Memories aren't made of this: amnesia at the movies. Br Med J. 2004;329(December):1480-3.

23. McClure J. The Role of Causal Attributions in Public Misconceptions About Brain Injury. Rehabil Psychol. 2011;56(2):85-93.

24. Linden MA, Crothers IR, O’Neill SB, McCann JP. Reduced community integration in persons following traumatic brain injury, as measured on the Community Integration Measure: An exploratory analysis. Disabil Rehabil. 2005;

25. Stergiou-Kita M, Grigorovich A, Damianakis T, Le Dorze G, David C, Lemsky C, et al. The big sell: Managing stigma and workplace discrimination following moderate to severe brain injury. Work. 2017;57:245-58.

26. Ono M, Ownsworth T, Walters B. Preliminary investigation of misconceptions and expectations of the effects of traumatic brain injury and symptom reporting. Brain Inj. 2011;25(2):237-49.

27. Chapman RCG, Hudson JM. Beliefs about brain injury in Britain. Brain Inj. 2010;24(6):797-801.

28. Springer JA, Farmer JE, Bouman DE. Common Misconceptions about Traumatic Brain Injury Among Family Members and Rehabilitation Patients. J Head Trauma Rehabil. 1997;12(3):41-50.

29. McKinlay A, Buck K. Misconceptions about traumatic brain injury among educators: has anything changed over the last 20 years? Disabil Rehabil [Internet]. 2018 Jan 28;18.

30. Mckinlay A, Bishop A, McLellan T. Public knowledge of 'concussion'and the different terminology used to communicate about mild traumatic brain injury (MTBI). Brain Inj. 2011;25:761-766.

31. Farmer JE, Johnson-Gerard M. Unfortunately, few educators have had formal training 
in how. Rehabil Psychol. 1997;42(4):273-86.

32. McKendry Y, Ownsworth T, Bettens GF. Comparing accuracy of knowledge of functional effects of schizophrenia and brain injury. Psychiatry Res. 2014;219:225-7.

33. Sefton J. An examination of factors that influence knowledge of and reporting of head injuries in college football. (Doctoral dissertation, Central Connecticut State University, New Britain, USA.; 2003.

34. Simonds C.B. Development of a Questionnaire to Assess Knowledge and Attitudes about Concussion and Return to Play Criteria in College Athletes. (Doctoral Dissertation. Philadelphia, PA: La Salle University; 2004.

35. Rosenbaum AM, Arnett PA. The development of a survey to examine knowledge about and attitudes toward concussion in high-school students. J Clin Exp Neuropsychol. 2009;1-13.

36. Williams JM, Langdon JL, McMillan JL, Buckley TA. English professional football players concussion knowledge and attitude. J Sport Heal Sci [Internet]. 2016;5(2):197204. Available from: http://dx.doi.org/10.1016/j.jshs.2015.01.009

37. World Health Organization. Towards a common language for functioning, disability and health: ICF. World Health Organization. Geneva; 2002. Available from: http://www.who.int/classifications/icf/training/icfbeginnersguide.pdf

38. King MF, Bruner GC. Social desirability bias: A neglected aspect of validity testing. Psychol Mark. 2000;17(2):79-103.

39. Crowne DP, Marlowe D. A new scale of social desirability independent of psychopathology. J Consult Psychol. 1960;

40. O’Rourke C, Linden MA, Lohan M. Misconceptions about traumatic brain injury among probation services. Disabil Rehabil. 2018;40(10):1119-26.

41. De Iorio ML, Nolan SA, Teague S. The effect of education type on common 
misconceptions of Traumatic Brain Injury. Rehabil Psychol. 2017;62(4):516-24.

42. Multon K. Test-Retest Reliability. In: Salkind NJ, editor. Encyclopedia of Research Design. Thousand Oaks: Sage Publications, Inc.; 2012. p. 1496-8.

43. Thomas M, Jobse E. Towards Measuring Community Understanding of Traumatic Brain Injury: The Structure and Potential Utility of the Head Injury Knowledge Scale. Brain Impair. 2015;16(2):104-15.

44. Watson D, Clark LA, Tellegen A. Development and validation of brief measures of positive and negative affect: The PANAS scales. J Pers Soc Psychol. 1988;54(6):106370.

45. Linden MA, Crothers IR. Violent, caring, unpredictable: Public views on survivors of brain injury. Arch Clin Neuropsychol. 2006;21:763-70.

46. Linden MA, Redpath SJ. A comparative study of nursing attitudes towards young male survivors of brain injury: A questionnaire survey. Int J Nurs Stud [Internet]. 2011;48(1):62-9. Available from: http://dx.doi.org/10.1016/j.ijnurstu.2010.05.011

47. Redpath S, Linden MA. Attitudes towards behavioural versus organic acquisition of brain injury. Brain Inj. 2004;18(9):861-9.

48. SJ R, Williams WH, Hanna D, Linden MA, Yates P, Harris A. Healthcare professionals ' attitudes towards traumatic brain injury ( TBI ): The influence of profession , experience , aetiology and blame on. Brain Inj. 2010;24(6):802-11.

49. Kelly J, St Lawrence JS, Smith S, Hood H V., Cook DJ. Medical students' attitudes toward AIDS and homosexual patients. J Med Educ. 1987;62:549-56.

50. McClure J, Devlin ME, McDowall J, Wade K. Visible markers of brain injury influence attributions for adolescents’ behaviour. Brain Inj. 2006;20(10):1029-35.

51. Linden MA, Boylan AM. “To be accepted as normal”: Public understanding and misconceptions concerning survivors of brain injury. Brain Inj. 2010;24(4):642-50. 
Bryant - Misconceptions about TBI 
Table 1: 25-item Gouvier et al.(9) survey and item use in subsequent scales.

Item $\quad$ Surveys using individual items from the scale

Items adopted for new scale development

Domain: Seatbelts

1. Wearing seatbelts causes as many injuries as it prevents.

2. It is safer to be trapped inside a wreck than to be thrown clear.

3. You don't need seatbelts as long as you can brace yourself before a crash.

4. It is more important to use seatbelts on long trips than in driving around town.

\section{Domain: Brain Damage}

5. A head injury can cause brain damage even if the person is not knocked out.

6. Problem with speech, coordination, or walking are usually due to brain damage.

7. Whiplash injuries to the neck can cause brain damage even if there is no direct blow to the head.

8. Most people with brain damage are not fully aware of its effect on their behavior.

9. A little brain damage doesn't matter much, since people only use a part of their brains anyway.

10. Emotional problems after head injury are usually not related to brain damage.

11. Most people with brain damage look and act retarded.

Domain: Unconscious

12. When people are knocked unconscious, most wake up shortly with no lasting effects.

13. Even after several weeks in a coma, when people wake up, most recognize and speak to others right away.

BIMS (10), CM-TBI (28)

CM-TBI (28)

BIMS (10), CM-TBI (28)

CM-TBI (28)

CM-TBI (28)

BIMS (10)

BIMS (10)*

BIMS (10), CM-TBI (28)

BIMS (10), CM-TBI (28) 
14. People in a coma are usually not aware of what is happening around them.

Domain: Amnesia

15. After a head injury, people can forget who they are and not recognize others, but be normal in every other way.

16. Sometimes a second blow to the head can help a person remember things that were forgotten.

17. People with amnesia for events before the injury usually have trouble learning new things too.

18. People usually have more trouble remembering things that happen after an injury than remembering things from before.

Domain: Recovery

19. How quickly a person recovers depends mainly on how hard they work at recovering.

20. People who have had one head injury are more likely to have a second one.

21. A person who has recovered from a head injury is less able to withstand a second blow to the head.

22. Once a recovering person feels "back to normal", the recovery process is complete.

23. It is good advice to rest and remain inactive during recovery.

24. "No pain-no gain” is good advice for a recovering patient.

25. Complete recovery from a severe head injury is not possible, no matter how badly the person wants
BIMS (10), CM-TBI (28)

BIMS (10), CM-TBI (28)

BIMS (10)*, CM-TBI (28)

BIMS (10), CM-TBI (28)

BIMS (10)*, CM-TBI (28)

BIMS (10), CM-TBI (28)

BIMS (10), CM-TBI (28)

BIMS (10), CM-TBI (28)

BIMS (10)

BIMS (10)

BIMS (10), CM-TBI (28) to recover

*wording alteration from original item ** replicated wording alteration provided by Willer et al. (12). 
Table 2: Overview of TBI measures and evaluation of their psychometric properties

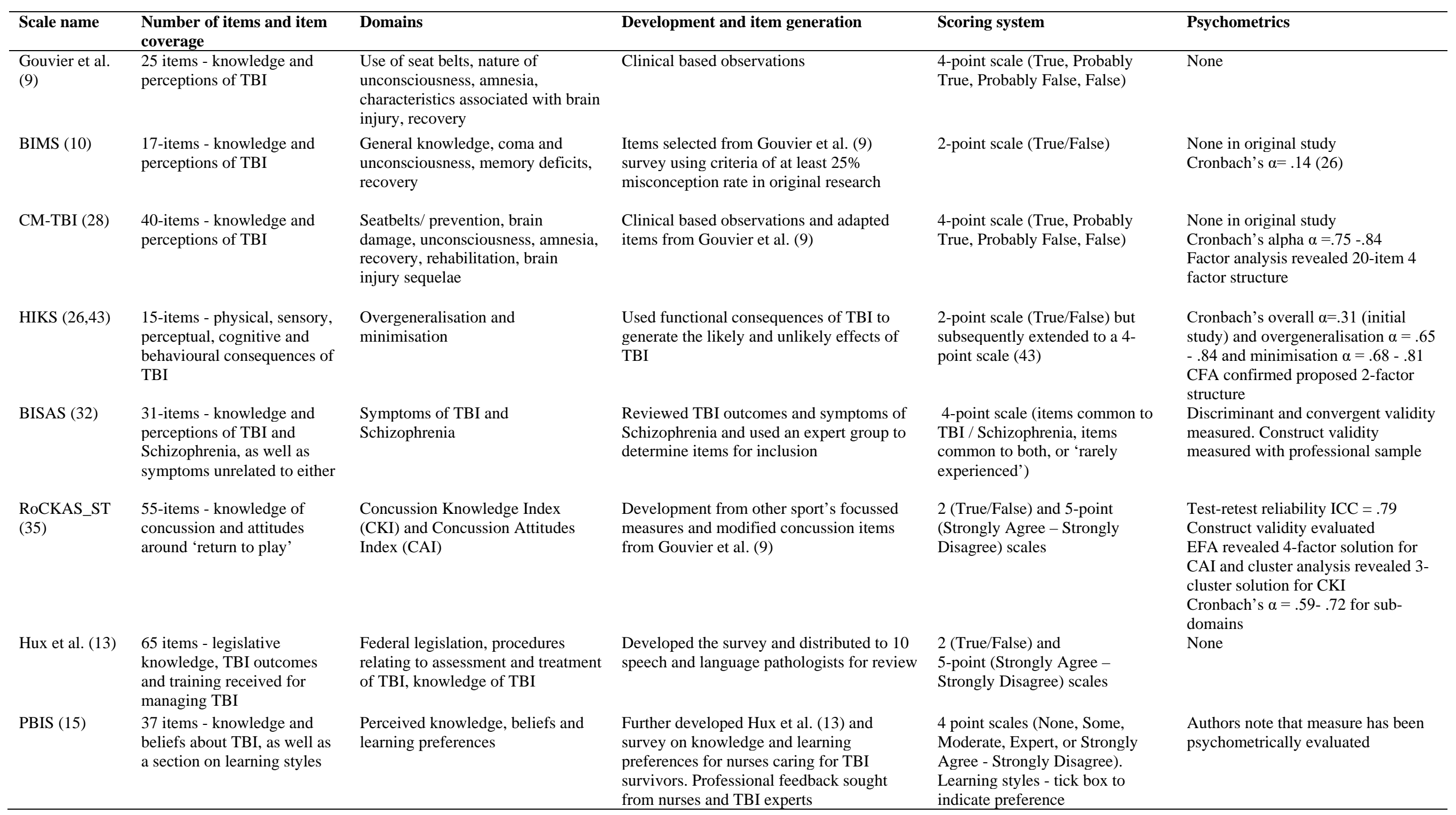

